\title{
Conversion of Hazardous Motor Vehicle Used Tire and Polystyrene Waste Plastic Mixture into useful Chemical Products
}

By

\author{
${ }^{1}$ Moinuddin Sarker and ${ }^{1}$ Mohammad Mamunor Rashid
}

\begin{abstract}
Motor vehicle used tire and polystyrene waste plastic mixture into fuel recovery using thermal degradation process in laboratory batch process. Motor vehicle used tire and polystyrene waste plastic was use $75 \mathrm{gm}$ by weight. Motor vehicle tire was $25 \mathrm{gm}$ and polystyrene waste plastic was $50 \mathrm{gm}$. In presence of oxygen experiment was performed under laboratory fume hood. Thermal degradation temperature range was $100-420{ }^{\circ} \mathrm{C}$ and experiment run time was 5 hours. Product fuel density is $0.84 \mathrm{gm} / \mathrm{ml}$ and liquid fuel conversion rate was $54.93 \%$. Fuel was analysis by GC/MS and compounds are present aliphatic group, aromatic group, alcoholic group, oxygen content and nitrogen content. Fuel can use refinery process as a refinery feed.
\end{abstract}

Keywords: Tire, polystyrene, conversion, chemical product, vehicle, hydrocarbon

\section{Introduction}

The generation of used tires in 2005 was estimated to be 2.5 million tonnes in North America, 2.5 million in Europe, and 0.5-1.0 million in Japan, which means $6 \mathrm{~kg}$ (approximately the weight of a car tire) per inhabitant and year in these developed countries [1]. The forecast for 2013 is that world generation will exceed 17 million tonnes per year, given that economic growth in developing countries drives vehicle sales and the substitution of less deteriorated tires, and the measures adopted to lengthen tire life are insufficient to offset these circumstances [2]. China generated 1 million tonnes in 2005 and the annual increase is $12 \%$. This outlook makes the valorization of used tires more interesting, and among the different technologies, pyrolysis has the following advantages: (i) it enables the subsequent individual valorization of gaseous, liquid, and carbon black fractions, which is an interesting aspect for economic viability; [3] (ii) it has 
a higher efficiency for energy and a lower environmental impact than incineration [4]. Different types of reactors have been used for tire pyrolysis, such as autoclaves[5] and fixed bed reactors, [6-10] and for a larger scale operation, bubbling fluidized bed reactors,[9-14] moving beds under vacuum, in one and two steps,[15-17] ablative beds,[18] and rotary ovens [19-21]. Key factors for process viability are high throughput and products with suitable properties for their subsequent valorization toward value added compounds (such as high-quality carbon black, active carbon, or chemical compounds, such as benzene, toluene, xylene, limonene, and so on) [22]. Pyrolysis as an attractive method to recycle scrap tires has recently been the subject of renewed interest. Pyrolysis of tires can produce oils, chars, and gases, in addition to the steel cords, all of which have the potential to be recycled. Within the past 2 decades, most experiments have been conducted using laboratory-scale batch units to characterize oil, char, and gas products [23].

Some conclusions from these laboratory-scale studies are as follows: Pyrolytic char has potential as a low-grade carbon black for a reinforcing filler or a printing ink pigment, [46] as a carbon adsorbent after proper activation, [7-9] and as a solid or slurry fuel [10]. Pyrolytic oil, a mixture of parafins, olefins, and aromatic compounds, possesses a high calorific value $(43 \mathrm{MJ} / \mathrm{kg})$ and can be used directly as fuel or can be added to petroleum refinery feedstocks [11-15]. Oils can also be properly cut based on their evaporating temperatures to solely produce valuable chemical feedstocks (i.e., benzene, xylene, toluene, and D-limonene), or some of the chemicals can be extracted with residue used as fuel [16-22]. Pyrolytic gas contains high concentrations of methane, butadiene, and other hydrocarbons, which results in a high calorific value $(35-40 \mathrm{MJ} / \mathrm{kg})$ sufficient to heat the pyrolysis reactor [23-25] The gas is generally not sold as a commercial product but used as a process heat resource because of its low yield (10 wt \%).

\section{Materials and Method}

\subsection{Materials}

Polystyrene waste plastic was collected from local restaurant and motor vehicle tire was collected from local car collision center. Both waste materials were washing with liquid soap and cut into small pieces. Waste Tire has metal portion and metal portion did put into reactor for liquefaction process. Laboratory experimental process sample was taken total $75 \mathrm{gm}$ by weight. $50 \mathrm{gm}$ of polystyrene waste and $25 \mathrm{gm}$ of motor vehicle used tire. Both waste materials have additives because plastic and tire manufacturing period manufacturing company are adding different types of additives for hardness and softness. Polystyrene plastic and motor vehicle tire chemical structure are shown figure 1. 


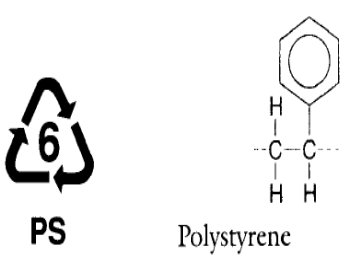

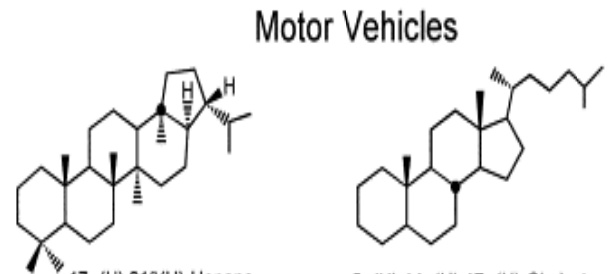

$5 \alpha(H), 14 \alpha(H), 17 \alpha(H)-$ Cholestane

Figure 1: Pobystyrene waste plastic structure and Tire structure

\subsection{Method Description}

Polystyrene waste plastic and motor vehicle used tire mixture into fuel production process setup shown into figure 2 . Small pieces waste materials put into reactor inside for liquefaction process. Then condensation unit was setup with reactor and collection device. Fuel collection container was setup with fuel purification system and final fuel collection container and sediment container. Gas cleaning device was setup with condensation unit with liquid solution (sodium hydroxide, sodium bicarbonate and water). Teflon bag, small pump was setup with cleaning device and residue collection container was connecting with reactor. Temperature range was $100-420{ }^{\circ} \mathrm{C}$ and thermal degradation without vacuumed system. No extra chemical was added in this experiment and whole experiment was close system. Experiment was monitored with temperature controller because some time experimental temperature need to increase and sometime experiment temperature need to decrease for quality product. Starting temperature 100 ${ }^{\circ} \mathrm{C}$ to final temperature $420^{\circ} \mathrm{C}$ was use for whole procedure finish. Total time consumes 5 hours and input electricity was $0.71 \mathrm{KWh}$. Waste polystyrene plastics and tire mixture to fuel production process during heating period was observed that lot of smoke was created. All vapors did not condense and came out as gas because it was light fraction $\mathrm{C}_{1}-\mathrm{C}_{4}$. Waste polystyrene plastic and tire has additives those additives cannot convert in to fuel only polymer portion can converted into fuel. Tire has high percentage of additives, cloth metal portion, and rubber and all of those parts cannot convertible. All additives come out as solid black residue. Polymer waste polystyrene has aromatic group and tire has petroleum component. When heat apply long chin carbon molecule breakdown and form into short chain hydrocarbon fuel or chemicals. Gas was cleaned with alkali wash and water wash then storage into Teflon bag using small pump. Fuel was filtered using RCI technology purification system. Product fuel density is $0.84 \mathrm{gm} / \mathrm{ml}$. In mass balance calculation showed $75 \mathrm{gm}$ polystyrene waste plastic and tire mixture to fuel weight is $41.2 \mathrm{gm}$, sample as light gas generated $2.5 \mathrm{gm}$, and leftover black residue was $31.3 \mathrm{gm}$. Conversion rate percentage result showed liquid fuel was $54.93 \%$, light gas generation $3.34 \%$ and residue percentage was $41.73 \%$. Residue percentage was high because tire has rubber and additive, cloth and metal. Residue and light gas analysis are under investigation. 


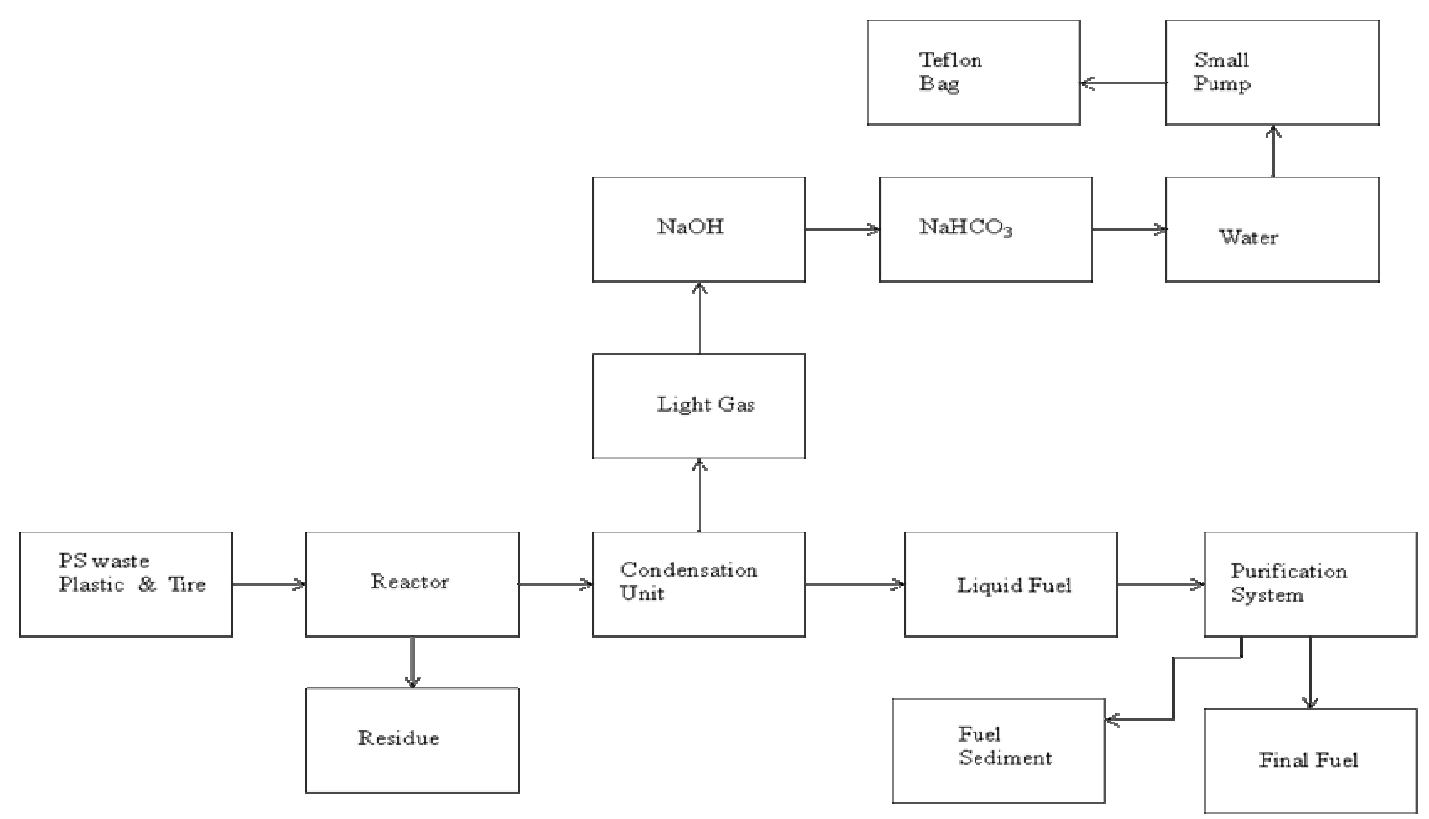

Figure 2: Polystyrene (PS) Waste Plastic and Motor Vebicle Used Tire to Chemical Product Production Process

\section{Results and Discussions}

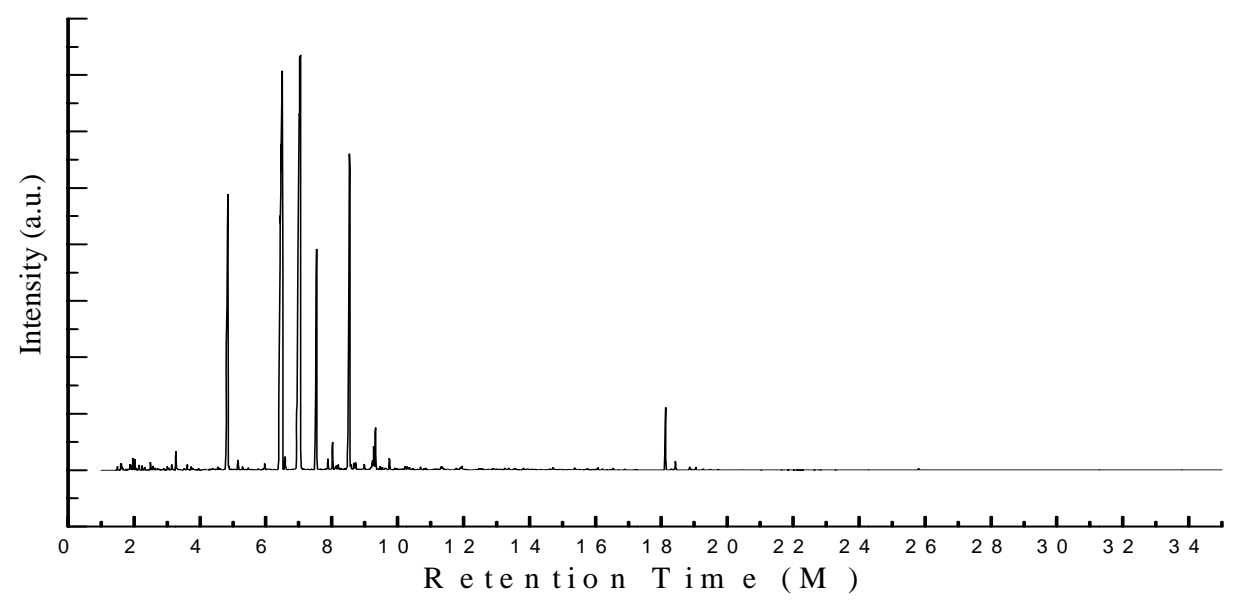

Figure 3: GC/MS Chromatogram of Polystyrene (PS) Waste Plastic and Motor Vehicle Used Tire to Chemical Product 
Table 1: GC/MS Chromatogram Compound List of Polystyrene (PS) Waste Plastic and Motor Vehicle Used Tire Mixture to Chemical Product

\begin{tabular}{|c|c|c|c|c|c|c|c|}
\hline $\begin{array}{l}\text { Numb } \\
\text { er of } \\
\text { Peak }\end{array}$ & $\begin{array}{l}\text { Retenti } \\
\text { on } \\
\text { Time } \\
\text { (min.) }\end{array}$ & $\begin{array}{l}\text { Trac } \\
\text { e } \\
\text { Mas } \\
\text { s } \\
(\mathrm{m} / \\
\text { z) }\end{array}$ & $\begin{array}{l}\text { Compound } \\
\text { Name }\end{array}$ & $\begin{array}{l}\text { Compo } \\
\text { und } \\
\text { Formul } \\
\text { a }\end{array}$ & $\begin{array}{l}\text { Molecu } \\
\text { lar } \\
\text { Weight }\end{array}$ & $\begin{array}{l}\text { Probabil } \\
\text { ity } \%\end{array}$ & $\begin{array}{l}\text { NIST } \\
\text { Library } \\
\text { Numb } \\
\text { er }\end{array}$ \\
\hline 1 & 1.49 & 41 & Cyclopropane & $\mathrm{C}_{3} \mathrm{H}_{6}$ & 42 & 40.9 & 18854 \\
\hline 2 & 1.56 & 43 & Isobutane & $\mathrm{C}_{4} \mathrm{H}_{10}$ & 58 & 68.9 & 18936 \\
\hline 3 & 1.60 & 41 & 2-Butene, (E)- & $\mathrm{C}_{4} \mathrm{H}_{8}$ & 56 & 23.9 & 105 \\
\hline 4 & 1.75 & 55 & 1-Butene, 3-methyl- & $\mathrm{C}_{5} \mathrm{H}_{10}$ & 70 & 23.6 & 160477 \\
\hline 5 & 1.81 & 43 & Butane, 2-methyl- & $\mathrm{C}_{5} \mathrm{H}_{12}$ & 72 & 77.4 & 19107 \\
\hline 6 & 1.87 & 42 & Cyclopropane, ethyl- & $\mathrm{C}_{5} \mathrm{H}_{10}$ & 70 & 39.6 & 114410 \\
\hline 7 & 1.91 & 55 & 1-Butanol, 3-methyl- & $\mathrm{O}^{\mathrm{C}_{5} \mathrm{H}_{12}}$ & 88 & 12.7 & 151656 \\
\hline 8 & 1.97 & 67 & 1,3-Pentadiene & $\mathrm{C}_{5} \mathrm{H}_{8}$ & 68 & 18.4 & 291890 \\
\hline 9 & 1.99 & 55 & $\begin{array}{l}\text { Cyclopropane, 1,2-dimethyl- } \\
\text {, trans- }\end{array}$ & $\mathrm{C}_{5} \mathrm{H}_{10}$ & 70 & 17.3 & 114453 \\
\hline 10 & 2.02 & 55 & 2-Pentene, (E)- & $\mathrm{C}_{5} \mathrm{H}_{10}$ & 70 & 18.0 & 291780 \\
\hline 11 & 2.06 & 67 & 1,4-Pentadiene & $\mathrm{C}_{5} \mathrm{H}_{8}$ & 68 & 27.0 & 114494 \\
\hline 12 & 2.25 & 67 & Bicyclo[2.1.0]pentane & $\mathrm{C}_{5} \mathrm{H}_{8}$ & 68 & 15.4 & 192491 \\
\hline 13 & 2.32 & 42 & 1-Pentanol, 2-methyl- & ${ }^{\mathrm{C}_{6} \mathrm{H}_{14}}$ & 102 & 29.5 & 19924 \\
\hline 14 & 2.44 & 57 & Pentane, 3-methyl- & $\mathrm{C}_{6} \mathrm{H}_{14}$ & 86 & 43.3 & 19375 \\
\hline 15 & 2.50 & 56 & 1-Hexene & $\mathrm{C}_{6} \mathrm{H}_{12}$ & 84 & 35.3 & 500 \\
\hline 16 & 2.57 & 57 & Hexane & $\mathrm{C}_{6} \mathrm{H}_{14}$ & 86 & 79.6 & 61280 \\
\hline 17 & 2.64 & 41 & Pentane, 3-methylene- & $\mathrm{C}_{6} \mathrm{H}_{12}$ & 84 & 27.3 & 19323 \\
\hline 18 & 2.68 & 41 & Pentane, 3-methylene- & $\mathrm{C}_{6} \mathrm{H}_{12}$ & 84 & 22.9 & 19323 \\
\hline 19 & 2.72 & 67 & $\begin{array}{l}\text { 1,3-Pentadiene, 3-methyl-, } \\
\text { (E)- }\end{array}$ & $\mathrm{C}_{6} \mathrm{H}_{10}$ & 82 & 9.33 & 62975 \\
\hline 20 & 2.78 & 41 & Pentane, 3-methylene- & $\mathrm{C}_{6} \mathrm{H}_{12}$ & 84 & 21.7 & 19323 \\
\hline 21 & 2.90 & 56 & Cyclopentane, methyl- & $\mathrm{C}_{6} \mathrm{H}_{12}$ & 84 & 55.0 & 114428 \\
\hline 22 & 2.96 & 67 & 3-Hexyne & $\mathrm{C}_{6} \mathrm{H}_{10}$ & 82 & 20.2 & 19282 \\
\hline 23 & 3.00 & 79 & $\begin{array}{l}\text { 1,3-Cyclopentadiene, } \\
\text { methyl- }\end{array}$ & $\mathrm{C}_{6} \mathrm{H}_{8}$ & 80 & 18.2 & 419 \\
\hline 24 & 3.14 & 67 & Cyclopentene, 1-methyl- & $\mathrm{C}_{6} \mathrm{H}_{10}$ & 82 & 12.3 & 107747 \\
\hline 25 & 3.27 & 78 & Benzene & $\mathrm{C}_{6} \mathrm{H}_{6}$ & 78 & 65.9 & 114388 \\
\hline 26 & 3.38 & 79 & $\begin{array}{l}\text { 1,3-Cyclopentadiene, } \\
\text { methyl- }\end{array}$ & $\mathrm{C}_{6} \mathrm{H}_{8}$ & 80 & 13.0 & 164279 \\
\hline 27 & 3.52 & 67 & Cyclohexene & $\mathrm{C}_{6} \mathrm{H}_{10}$ & 82 & 21.6 & 61209 \\
\hline 28 & 3.57 & 56 & $\begin{array}{l}\text { Cyclobutanone, } \\
\text { dimethyl- }\end{array}$ & $\mathrm{O}^{\mathrm{C}_{6} \mathrm{H}_{10}}$ & 98 & 24.1 & 957 \\
\hline 29 & 3.62 & 41 & 1-Heptene & $\mathrm{C}_{7} \mathrm{H}_{14}$ & 98 & 27.9 & 19704 \\
\hline 30 & 3.74 & 43 & Heptane & $\mathrm{C}_{7} \mathrm{H}_{16}$ & 100 & 51.3 & 61276 \\
\hline 31 & 3.78 & 81 & $\begin{array}{l}\text { Cyclopropane, } \\
\text { trimethylmethylene- }\end{array}$ & $\mathrm{C}_{7} \mathrm{H}_{12}$ & 96 & 14.1 & 63085 \\
\hline 32 & 3.96 & 81 & Cyclopentene, 4,4-dimethyl- & $\mathrm{C}_{7} \mathrm{H}_{12}$ & 96 & 10.3 & 38642 \\
\hline 33 & 4.09 & 67 & 1-Ethylcyclopentene & $\mathrm{C}_{7} \mathrm{H}_{12}$ & 96 & 33.7 & 114407 \\
\hline 34 & 4.16 & 55 & Cyclohexane, methyl- & $\mathrm{C}_{7} \mathrm{H}_{14}$ & 98 & 41.1 & 118503 \\
\hline 35 & 4.21 & 79 & 1,3,5-Heptatriene, (E,E)- & $\mathrm{C}_{7} \mathrm{H}_{10}$ & 94 & 7.59 & 118126 \\
\hline 36 & 4.31 & 43 & Acetic acid, dichloro-, heptyl & $\mathrm{C}_{9} \mathrm{H}_{16}$ & 226 & 7.15 & 280485 \\
\hline
\end{tabular}




\begin{tabular}{|c|c|c|c|c|c|c|c|}
\hline & & & ester & $\mathrm{Cl}_{22}$ & & & \\
\hline 37 & 4.39 & 79 & 1,3-Cycloheptadiene & $\mathrm{C}_{7} \mathrm{H}_{10}$ & 94 & 10.4 & 237922 \\
\hline 38 & 4.50 & 79 & $\begin{array}{l}\text { 1,3-Cyclopentadiene, } \\
\text { dimethyl- }\end{array}$ & $\mathrm{C}_{7} \mathrm{H}_{10}$ & 94 & 10.3 & 800 \\
\hline 39 & 4.55 & 81 & $\begin{array}{l}\text { Cyclobutane, } \\
\text { methylethylidene)- }\end{array}$ & $\mathrm{C}_{7} \mathrm{H}_{12}$ & 96 & 11.1 & 150272 \\
\hline 40 & 4.61 & 67 & Cyclopentane, ethylidene- & $\mathrm{C}_{7} \mathrm{H}_{12}$ & 96 & 35.1 & 114403 \\
\hline 41 & 4.85 & 91 & Toluene & $\mathrm{C}_{7} \mathrm{H}_{8}$ & 92 & 56.6 & 291301 \\
\hline 42 & 5.05 & 55 & $\begin{array}{l}\text { (5-Methylcyclopent-1- } \\
\text { enyl)methanol }\end{array}$ & $\mathrm{O}^{\mathrm{C}_{7} \mathrm{H}_{12}}$ & 112 & 22.9 & 99174 \\
\hline 43 & 5.15 & 55 & Pentane, 2-cyclopropyl- & $\mathrm{C}_{8} \mathrm{H}_{16}$ & 112 & 9.93 & 113439 \\
\hline 44 & 5.23 & 55 & 2-Octyn-1-ol & $\mathrm{C}_{8} \mathrm{H}_{14}$ & 126 & 25.1 & 113247 \\
\hline & & & & $\mathrm{O}$ & & & \\
\hline 45 & 5.29 & 43 & Octane & $\mathrm{C}_{8} \mathrm{H}_{18}$ & 114 & 36.4 & 61242 \\
\hline 46 & 5.39 & 55 & 3-Octene, (Z)- & $\mathrm{C}_{8} \mathrm{H}_{16}$ & 112 & 12.7 & 113895 \\
\hline 47 & 5.47 & 207 & $\begin{array}{l}\text { Cyclotrisiloxane, } \\
\text { hexamethyl- }\end{array}$ & $\begin{array}{l}\mathrm{C}_{6} \mathrm{H}_{18} \\
\mathrm{O}_{3} \mathrm{Si}_{3}\end{array}$ & 222 & 72.7 & 238029 \\
\hline 48 & 5.55 & 93 & Pyridine, 3-methyl- & $\mathrm{C}_{6} \mathrm{H}_{7} \mathrm{~N}$ & 93 & 50.8 & 791 \\
\hline 49 & 5.65 & 81 & 3,5-Octadiene, (Z,Z)- & $\mathrm{C}_{8} \mathrm{H}_{14}$ & 110 & 12.7 & 250525 \\
\hline 50 & 5.77 & 95 & $\begin{array}{l}\text { 1-Methyl-2- } \\
\text { methylenecyclohexane }\end{array}$ & $\mathrm{C}_{8} \mathrm{H}_{14}$ & 110 & 9.50 & 113437 \\
\hline 51 & 5.92 & 81 & $\begin{array}{l}\text { 1-Ethyl-5- } \\
\text { methylcyclopentene }\end{array}$ & $\mathrm{C}_{8} \mathrm{H}_{14}$ & 110 & 17.1 & 114420 \\
\hline 52 & 5.97 & 54 & Cyclohexene, 4-ethenyl- & $\mathrm{C}_{8} \mathrm{H}_{12}$ & 108 & 28.9 & 227540 \\
\hline 53 & 6.05 & 93 & $\begin{array}{l}\text { Cyclohexane, } \\
\text { bis(methylene)- }\end{array}$ & $\mathrm{C}_{8} \mathrm{H}_{12}$ & 108 & 14.4 & 1399 \\
\hline 54 & 6.46 & 106 & Ethylbenzene & $\mathrm{C}_{8} \mathrm{H}_{10}$ & 106 & 39.8 & 158804 \\
\hline 55 & 6.58 & 91 & p-Xylene & $\mathrm{C}_{8} \mathrm{H}_{10}$ & 106 & 30.4 & 113952 \\
\hline 56 & 7.05 & 103 & 1,3,5,7-Cyclooctatetraene & $\mathrm{C}_{8} \mathrm{H}_{8}$ & 104 & 38.6 & 113230 \\
\hline 57 & 7.54 & 105 & Benzene, (1-methylethyl)- & $\mathrm{C}_{9} \mathrm{H}_{12}$ & 120 & 42.5 & 228742 \\
\hline 58 & 7.88 & 117 & Benzene, 2-propenyl- & $\mathrm{C}_{9} \mathrm{H}_{10}$ & 118 & 16.1 & 114744 \\
\hline 59 & 8.02 & 91 & Benzene, propyl- & $\mathrm{C}_{9} \mathrm{H}_{12}$ & 120 & 75.9 & 113930 \\
\hline 60 & 8.14 & 105 & Benzene, 1-ethyl-3-methyl- & $\mathrm{C}_{9} \mathrm{H}_{12}$ & 120 & 35.2 & 228743 \\
\hline 61 & 8.20 & 105 & Benzene, 1-ethyl-3-methyl- & $\mathrm{C}_{9} \mathrm{H}_{12}$ & 120 & 29.0 & 228743 \\
\hline 62 & 8.28 & 105 & $\begin{array}{l}\text { 2,3-Heptadien-5-yne, } \\
\text { dimethyl- }\end{array}$ & $\mathrm{C}_{9} \mathrm{H}_{12}$ & 120 & 7.80 & 33204 \\
\hline 63 & 8.54 & 117 & $\alpha$-Methylstyrene & $\mathrm{C}_{9} \mathrm{H}_{10}$ & 118 & 34.3 & 2021 \\
\hline 64 & 8.73 & 105 & Benzene, 1,3,5-trimethyl- & $\mathrm{C}_{9} \mathrm{H}_{12}$ & 120 & 19.7 & 20469 \\
\hline 65 & 8.99 & 105 & Benzene, (1-methylpropyl)- & $\mathrm{C}_{10} \mathrm{H}_{14}$ & 134 & 38.4 & 228188 \\
\hline 66 & 9.23 & 119 & $\begin{array}{l}\text { Benzene, 1-methyl-3-(1- } \\
\text { methylethyl)- }\end{array}$ & $\mathrm{C}_{10} \mathrm{H}_{14}$ & 134 & 16.6 & 149866 \\
\hline 67 & 9.27 & 117 & Benzene, 2-propenyl- & $\mathrm{C}_{9} \mathrm{H}_{10}$ & 118 & 16.8 & 114744 \\
\hline 68 & 9.33 & 68 & Limonene & $\mathrm{C}_{10} \mathrm{H}_{16}$ & 136 & 21.0 & 57640 \\
\hline 69 & 9.46 & 117 & Indane & $\mathrm{C}_{9} \mathrm{H}_{10}$ & 118 & 13.1 & 118485 \\
\hline 70 & 9.53 & 91 & Benzene, 3-butenyl- & $\mathrm{C}_{10} \mathrm{H}_{12}$ & 132 & 76.5 & 113933 \\
\hline 71 & 9.63 & 115 & Benzene, 1-propynyl- & $\mathrm{C}_{9} \mathrm{H}_{8}$ & 116 & 25.4 & 113196 \\
\hline 72 & 9.75 & 91 & $\begin{array}{l}\text { 1,2,3,4,5,8- } \\
\text { Hexahydronaphthalene }\end{array}$ & $\mathrm{C}_{10} \mathrm{H}_{14}$ & 134 & 25.2 & 113559 \\
\hline 73 & 9.92 & 117 & $\begin{array}{l}\text { Benzene, } \\
\text { propenyl)- }\end{array}$ & $\mathrm{C}_{10} \mathrm{H}_{12}$ & 132 & 17.7 & 113549 \\
\hline 74 & 10.22 & 41 & 3-Undecene, (E)- & $\mathrm{C}_{11} \mathrm{H}_{22}$ & 154 & 4.78 & 60565 \\
\hline 75 & 10.29 & 117 & 2,4-Dimethylstyrene & $\mathrm{C}_{10} \mathrm{H}_{12}$ & 132 & 10.6 & 136251 \\
\hline 76 & 10.36 & 57 & Undecane & $\mathrm{C}_{11} \mathrm{H}_{24}$ & 156 & 24.1 & 114185 \\
\hline 77 & 10.46 & 105 & Benzene, (1-methylbutyl)- & $\mathrm{C}_{11} \mathrm{H}_{16}$ & 148 & 35.8 & 245071 \\
\hline
\end{tabular}




\begin{tabular}{|c|c|c|c|c|c|c|c|}
\hline 78 & 10.70 & 117 & 1-methyl-4-(2- & $\mathrm{C}_{10} \mathrm{H}_{12}$ & 132 & 13.1 & 113549 \\
\hline 79 & 10.86 & 117 & $\begin{array}{l}\text { Benzene, } \\
\text { propenyl)- }\end{array}$ & $\mathrm{C}_{10} \mathrm{H}_{12}$ & 132 & 11.4 & 113536 \\
\hline 80 & 11.32 & 115 & $\begin{array}{l}\text { 4-Methyl- } \alpha \text {-methyl- } \alpha \text { - } \\
\text { nitrostyrene }\end{array}$ & $\begin{array}{l}\mathrm{C}_{10} \mathrm{H}_{11} \\
\mathrm{NO}_{2}\end{array}$ & 177 & 7.20 & 135064 \\
\hline 81 & 11.35 & 91 & Benzene, pentyl- & $\mathrm{C}_{11} \mathrm{H}_{16}$ & 148 & 69.7 & 113915 \\
\hline 82 & 11.78 & 41 & 3-Dodecene, (E)- & $\mathrm{C}_{12} \mathrm{H}_{24}$ & 168 & 9.07 & 113960 \\
\hline 83 & 11.90 & 57 & Dodecane & $\mathrm{C}_{12} \mathrm{H}_{26}$ & 170 & 8.59 & 291499 \\
\hline 84 & 11.94 & 128 & Naphthalene & $\mathrm{C}_{10} \mathrm{H}_{8}$ & 128 & 23.5 & 114935 \\
\hline 85 & 12.02 & 131 & $\begin{array}{l}\text { Benzene, 1-methyl-3-(1- } \\
\text { methyl-2-propenyl)- }\end{array}$ & $\mathrm{C}_{11} \mathrm{H}_{14}$ & 146 & 13.4 & 155781 \\
\hline 86 & 12.38 & 117 & trans-1-Phenyl-1-pentene & $\mathrm{C}_{11} \mathrm{H}_{14}$ & 146 & 19.2 & 113579 \\
\hline 87 & 12.47 & 117 & Benzene, cyclopentyl- & $\mathrm{C}_{11} \mathrm{H}_{14}$ & 146 & 24.8 & 187011 \\
\hline 88 & 12.54 & 135 & Benzothiazole & $\mathrm{C}_{7} \mathrm{H}_{5} \mathrm{~N}$ & 135 & 65.7 & 73061 \\
\hline & & & & S & & & \\
\hline 89 & 12.89 & 91 & Benzene, hexyl- & $\mathrm{C}_{12} \mathrm{H}_{18}$ & 162 & 50.0 & 113954 \\
\hline 90 & 13.01 & 129 & Benzene, 4-pentynyl- & $\mathrm{C}_{11} \mathrm{H}_{12}$ & 144 & 16.6 & 113572 \\
\hline 91 & 13.25 & 41 & 7-Tetradecene & $\mathrm{C}_{14} \mathrm{H}_{28}$ & 196 & 7.77 & 70643 \\
\hline 92 & 13.36 & 57 & Tridecane & $\mathrm{C}_{13} \mathrm{H}_{28}$ & 184 & 13.9 & 114282 \\
\hline 93 & 13.55 & 144 & $\begin{array}{l}\text { 2,3-Diazabicyclo[2.2.1]hept- } \\
\text { 2-ene, 5-phenyl- }\end{array}$ & $\begin{array}{l}\mathrm{C}_{11} \mathrm{H}_{12} \\
\mathrm{~N}_{2}\end{array}$ & 172 & 18.0 & 142358 \\
\hline 94 & 13.82 & 142 & 1H-Indene, 1-ethylidene- & $\mathrm{C}_{11} \mathrm{H}_{10}$ & 142 & 22.6 & 155753 \\
\hline 95 & 14.06 & 104 & Benzene, 3-cyclohexen-1-yl- & $\mathrm{C}_{12} \mathrm{H}_{14}$ & 158 & 62.8 & 114816 \\
\hline 96 & 14.35 & 91 & Benzene, heptyl- & $\mathrm{C}_{13} \mathrm{H}_{20}$ & 176 & 49.3 & 60570 \\
\hline 97 & 14.45 & 104 & $\begin{array}{l}\text { (4-Methyl-1-methylenepent- } \\
\text { 4-enyl)benzene }\end{array}$ & $\mathrm{C}_{13} \mathrm{H}_{16}$ & 172 & 17.7 & 210729 \\
\hline 98 & 14.62 & 41 & 7-Tetradecene & $\mathrm{C}_{14} \mathrm{H}_{28}$ & 196 & 10.7 & 70643 \\
\hline 99 & 14.71 & 154 & Biphenyl & $\mathrm{C}_{12} \mathrm{H}_{10}$ & 154 & 66.4 & 53609 \\
\hline 100 & 14.90 & 80 & $\begin{array}{l}\text { Benzene, } \\
\text { methylbutylidene)bis- }\end{array}$ & $\mathrm{C}_{17} \mathrm{H}_{20}$ & 224 & 13.4 & 10579 \\
\hline 101 & 15.21 & 80 & 17-Octadecen-14-yn-1-ol & $\mathrm{O}^{\mathrm{C}_{18} \mathrm{H}_{32}}$ & 264 & 8.14 & 36083 \\
\hline 102 & 15.37 & 167 & Diphenylmethane & $\mathrm{C}_{13} \mathrm{H}_{12}$ & 168 & 50.7 & 21969 \\
\hline 103 & 15.52 & 157 & Quinoline, 4,8-dimethyl- & $\mathrm{C}_{11} \mathrm{H}_{11}$ & 157 & 28.7 & 5413 \\
\hline 104 & 15.76 & 94 & $\begin{array}{l}\text { 1,7-Dimethyl-4-oxa- } \\
\text { tricyclo[5.2.1.0(2,6)]decane- } \\
\text { 3,5,8-trione }\end{array}$ & ${ }_{\mathrm{O}_{4}}^{\mathrm{N}} \mathrm{C}_{11} \mathrm{H}_{12}$ & 208 & 11.5 & 275441 \\
\hline 105 & 16.02 & 57 & Tetradecane & $\mathrm{C}_{14} \mathrm{H}_{30}$ & 198 & 15.2 & 113925 \\
\hline 106 & 16.08 & 167 & Benzene, 1,1'-ethylidenebis- & $\mathrm{C}_{14} \mathrm{H}_{14}$ & 182 & 43.1 & 22224 \\
\hline 107 & 16.21 & 168 & 1,1'-Biphenyl, 4-methyl- & $\mathrm{C}_{13} \mathrm{H}_{12}$ & 168 & 21.3 & 113287 \\
\hline 108 & 16.54 & 91 & $\begin{array}{l}\begin{array}{l}\text { Benzene, } \\
\text { ethanediyl }) \text { bis- }\end{array} \\
\text { 1,1'-(1,2- }\end{array}$ & $\mathrm{C}_{14} \mathrm{H}_{14}$ & 182 & 87.2 & 187213 \\
\hline 109 & 16.70 & 170 & $\begin{array}{l}\text { 3-(2-Methyl-propenyl)-1H- } \\
\text { indene }\end{array}$ & $\mathrm{C}_{13} \mathrm{H}_{14}$ & 170 & 32.7 & 187785 \\
\hline 110 & 16.89 & 105 & $\begin{array}{l}\text { Benzene, 1,1'-(1-methyl-1,2- } \\
\text { ethanediyl)bis- }\end{array}$ & $\mathrm{C}_{15} \mathrm{H}_{16}$ & 196 & 39.0 & 34633 \\
\hline 111 & 17.01 & 91 & Benzene, nonyl- & $\mathrm{C}_{15} \mathrm{H}_{24}$ & 204 & 5.77 & 113903 \\
\hline 112 & 17.16 & 41 & $\begin{array}{l}\text { Cyclohexane, 2-butyl-1,1,3- } \\
\text { trimethyl- }\end{array}$ & $\mathrm{C}_{13} \mathrm{H}_{26}$ & 182 & 15.3 & 7582 \\
\hline 113 & 17.24 & 57 & Hexadecane & $\mathrm{C}_{16} \mathrm{H}_{34}$ & 226 & 9.89 & 62249 \\
\hline 114 & 17.30 & 105 & Benzene, 1,1'-(1,2-dimethyl- & $\mathrm{C}_{16} \mathrm{H}_{18}$ & 210 & 10.1 & 41226 \\
\hline
\end{tabular}




\begin{tabular}{|c|c|c|c|c|c|c|c|}
\hline & & & $\begin{array}{l}\text { 1,2-ethanediyl)bis-, } \quad\left(\mathrm{R}^{*}, \mathrm{R}^{*}\right)- \\
(\tilde{\mathrm{n}}) \text { - }\end{array}$ & & & & \\
\hline 115 & 17.43 & 167 & Benzene, 1,1'-ethylidenebis- & $\mathrm{C}_{14} \mathrm{H}_{14}$ & 182 & 36.9 & 22224 \\
\hline 116 & 17.83 & 44 & $\begin{array}{l}\text { Benzene, } \\
\text { (phenylmethyl)- }\end{array}$ & $\mathrm{C}_{14} \mathrm{H}_{14}$ & 182 & 36.4 & 202278 \\
\hline 117 & 18.13 & 92 & $\begin{array}{l}\text { Benzene, } \\
\text { propanediyl)bis- }\end{array}$ & $\mathrm{C}_{15} \mathrm{H}_{16}$ & 196 & 95.7 & 133399 \\
\hline 118 & 18.43 & 105 & $\begin{array}{l}\text { Benzene, 1,1'-(1-methyl-1,3- } \\
\text { propanediyl)bis- }\end{array}$ & $\mathrm{C}_{16} \mathrm{H}_{18}$ & 210 & 90.5 & 149665 \\
\hline 119 & 18.86 & 91 & $\begin{array}{l}\text { Naphthalene, } \\
\text { tetrahydro-2-phenyl- }\end{array}$ & $\mathrm{C}_{16} \mathrm{H}_{16}$ & 208 & 29.6 & 9510 \\
\hline 120 & 19.47 & 115 & $\begin{array}{l}\text { Benzene, } \\
\text { butenylidene)bis- }\end{array}$ & $\mathrm{C}_{16} \mathrm{H}_{16}$ & 208 & 23.8 & 156823 \\
\hline 121 & 19.73 & 115 & $\begin{array}{l}\text { Benzene, 1,1'-(3-methyl-1- } \\
\text { propene-1,3-diyl)bis- }\end{array}$ & $\mathrm{C}_{16} \mathrm{H}_{16}$ & 208 & 48.1 & 9505 \\
\hline 122 & 21.15 & 44 & 1-Pentene, 1,5-diphenyl- & $\mathrm{C}_{17} \mathrm{H}_{18}$ & 222 & 22.5 & 63202 \\
\hline 123 & 25.80 & 91 & $\begin{array}{l}(2,3- \\
\text { Diphenylcyclopropyl)methyl } \\
\text { phenyl sulfoxide, trans- }\end{array}$ & $\begin{array}{l}\mathrm{C}_{22} \mathrm{H}_{20} \\
\mathrm{OS}\end{array}$ & 332 & 26.5 & 142947 \\
\hline
\end{tabular}

Motor vehicle waste tire and polystyrene waste plastic mixture to product fuel was analysis by GC/MS and determine compounds structure. Figure 3 and table 1 shown analysis GC/MS chromatogram and chromatogram compounds list. Perkin Elmer GC/MS (model clarus 500) was use for liquid fuel analysis purpose. Chromatogram compounds was detected form chromatogram based on trace mass $(\mathrm{m} / \mathrm{z})$ and retention time (t). Compounds table showed product fuel has large amount of aromatic group and aliphatic group. Oxygen content, nitrogen content, sulfur and halogenated group are present in this fuel. GC/MS analysis result showed chromatogram starting compound is Cyclopropane $\left(\mathrm{C}_{3} \mathrm{H}_{6}\right)(\mathrm{t}=1.49, \mathrm{~m} / \mathrm{z}=41)$ and highest carbon chain compound is trans(2, 3-Diphenylcyclopropyl) methyl phenyl sulfoxide $\left(\mathrm{C}_{22} \mathrm{H}_{20} \mathrm{OS}\right)(\mathrm{t}=25.80, \mathrm{~m} / \mathrm{z}=91)$. All compounds were traced based on retention time, trace mass and peak intensity. For compounds detection purpose search library was use Perkin Elmer NIST library. Compounds were traced lower number carbon compounds to higher number carbon compounds such as Isobutane $\left(\mathrm{C}_{4} \mathrm{H}_{10}\right)(\mathrm{t}=1.56, \mathrm{~m} / \mathrm{z}=43)$ Compound probability percentage is $68.9 \%$, 2-methyl- Butane $\left(\mathrm{C}_{5} \mathrm{H}_{12}\right) \quad(\mathrm{t}=1.81, \mathrm{~m} / \mathrm{z}=43)$ Compound probability percentage is $77.4 \%$, trans-1, 2-dimethyl- Cyclopropane $\left(\mathrm{C}_{5} \mathrm{H}_{10}\right)(\mathrm{t}=1.99$, $\mathrm{m} / \mathrm{z}=55)$ Compound probability percentage is $17.3 \%$, 3-methyl-Pentane $\left(\mathrm{C}_{6} \mathrm{H}_{14}\right)$ $(\mathrm{t}=2.44, \mathrm{~m} / \mathrm{z}=57)$ Compound probability percentage is $43.3 \%$, 3-methylene-Pentane $\left(\mathrm{C}_{6} \mathrm{H}_{12}\right) \quad(\mathrm{t}=2.68, \mathrm{~m} / \mathrm{z}=41)$ Compound probability percentage is $22.9 \%$, methylCyclopentane $\left(\mathrm{C}_{6} \mathrm{H}_{12}\right)(\mathrm{t}=2.90, \mathrm{~m} / \mathrm{z}=56)$ Compound probability percentage is $55.0 \%$, Benzene $\left(\mathrm{C}_{6} \mathrm{H}_{6}\right)(\mathrm{t}=3.27, \mathrm{~m} / \mathrm{z}=78)$ Compound probability percentage is $65.9 \%, 3,3-$ dimethyl-Cyclobutanone $\left(\mathrm{C}_{6} \mathrm{H}_{10} \mathrm{O}\right) \quad(\mathrm{t}=3.57, \mathrm{~m} / \mathrm{z}=56)$ Compound probability percentage is $24.1 \%$, trimethylmethylene-Cyclopropane $\left(\mathrm{C}_{7} \mathrm{H}_{12}\right)(\mathrm{t}=3.78, \mathrm{~m} / \mathrm{z}=81)$ Compound probability percentage is $14.1 \%$, methyl-Cyclohexane $\left(\mathrm{C}_{7} \mathrm{H}_{14}\right) \quad(\mathrm{t}=4.16$, $\mathrm{m} / \mathrm{z}=55)$ Compound probability percentage is $41.1 \%$, 1,2-dimethyl-1,3-Cyclopentadiene $\left(\mathrm{C}_{7} \mathrm{H}_{10}\right)(\mathrm{t}=4.50, \mathrm{~m} / \mathrm{z}=79)$ Compound probability percentage is $10.3 \%$, Toluene $\left(\mathrm{C}_{7} \mathrm{H}_{8}\right)$ 
$(\mathrm{t}=4.85, \mathrm{~m} / \mathrm{z}=91)$ Compound probability percentage is $56.6 \%, 2$-Octyn-1-ol $\left(\mathrm{C}_{8} \mathrm{H}_{14} \mathrm{O}\right)$ $(\mathrm{t}=5.23, \mathrm{~m} / \mathrm{z}=55)$ Compound probability percentage is $25.1 \%$, 3-methyl- Pyridine $\left(\mathrm{C}_{6} \mathrm{H}_{7} \mathrm{~N}\right)(\mathrm{t}=5.55, \mathrm{~m} / \mathrm{z}=93)$ Compound probability percentage is $50.8 \%$, 1-Ethyl-5methylcyclopentene $\left(\mathrm{C}_{8} \mathrm{H}_{14}\right)(\mathrm{T}=5.92, \mathrm{~m} / \mathrm{z}=81)$ Compound probability percentage is $17.1 \%$, Ethylbenzene $\left(\mathrm{C}_{8} \mathrm{H}_{10}\right)(\mathrm{t}=6.46, \mathrm{~m} / \mathrm{z}=106)$ Compound probability percentage is $39.8 \%$, 2-propenyl- Benzene $\left(\mathrm{C}_{9} \mathrm{H}_{10}\right) \quad(\mathrm{t}=7.88, \mathrm{~m} / \mathrm{z}=117)$ Compound probability percentage is $16.1 \%$, 1-ethyl-3-methyl- Benzene $\left(\mathrm{C}_{9} \mathrm{H}_{12}\right)(\mathrm{t}=8.20, \mathrm{~m} / \mathrm{z}=105)$ Compound probability percentage is $29.0 \%, \alpha$-Methylstyrene $\left(\mathrm{C}_{9} \mathrm{H}_{10}\right) \quad(\mathrm{t}=8.54, \mathrm{~m} / \mathrm{z}=117)$ Compound probability percentage is $34.3 \%$, 2-propenyl- Benzene $\left(\mathrm{C}_{9} \mathrm{H}_{10}\right)(\mathrm{t}=9.27$, $\mathrm{m} / \mathrm{z}=117)$ Compound probability percentage is $16.8 \%$, Limonene $\left(\mathrm{C}_{10} \mathrm{H}_{16}\right)(\mathrm{t}=9.33$, $\mathrm{m} / \mathrm{z}=68$ ) Compound probability percentage is $21.0 \%$, 1-propynyl- Benzene $\left(\mathrm{C}_{9} \mathrm{H}_{8}\right)$ $(\mathrm{t}=9.63, \mathrm{~m} / \mathrm{z}=115)$ Compound probability percentage is $25.4 \%$, Undecane $\left(\mathrm{C}_{11} \mathrm{H}_{24}\right)$ $(\mathrm{t}=10.36, \mathrm{~m} / \mathrm{z}=57)$ Compound probability percentage is $24.1 \%$, (2-methyl-2-propenyl)Benzene $\left(\mathrm{C}_{10} \mathrm{H}_{12}\right)(\mathrm{t}=10.86, \mathrm{~m} / \mathrm{z}=117)$ Compound probability percentage is $11.4 \%$, (E)-3-Dodecene $\left(\mathrm{C}_{12} \mathrm{H}_{24}\right)(\mathrm{t}=11.78, \mathrm{~m} / \mathrm{z}=41)$ Compound probability percentage is $9.07 \%$, Naphthalene $\left(\mathrm{C}_{10} \mathrm{H}_{8}\right)(\mathrm{t}=11.94, \mathrm{~m} / \mathrm{z}=128)$ Compound probability percentage is $23.5 \%$, cyclopentyl-Benzene $\left(\mathrm{C}_{11} \mathrm{H}_{14}\right) \quad(\mathrm{t}=12.47, \mathrm{~m} / \mathrm{z}=117)$ Compound probability percentage is $24.8 \%$, Tridecane $\left(\mathrm{C}_{13} \mathrm{H}_{28}\right)(\mathrm{t}=13.36, \mathrm{~m} / \mathrm{z}=57)$ Compound probability percentage is $13.9 \%$, 3-cyclohexen-1-yl- Benzene $\left(\mathrm{C}_{12} \mathrm{H}_{14}\right) \quad(\mathrm{t}=14.06, \mathrm{~m} / \mathrm{z}=104)$ Compound probability percentage is $62.8 \%$, Biphenyl $\left(\mathrm{C}_{12} \mathrm{H}_{10}\right)(\mathrm{t}=14.71, \mathrm{~m} / \mathrm{z}=154)$ Compound probability percentage is $66.4 \%$, Diphenylmethane $\left(\mathrm{C}_{13} \mathrm{H}_{12}\right) \quad(\mathrm{t}=15.37$, $\mathrm{m} / \mathrm{z}=167)$ Compound probability percentage is $50.7 \%$, Tetradecane $\left(\mathrm{C}_{14} \mathrm{H}_{30}\right)(\mathrm{t}=16.02$, $\mathrm{m} / \mathrm{z}=57)$ Compound probability percentage is $15.2 \%$, bis-1,1'-(1,2-ethanediyl) Benzene $\left(\mathrm{C}_{14} \mathrm{H}_{14}\right)(\mathrm{t}=16.54, \mathrm{~m} / \mathrm{z}=91)$ Compound probability percentage is $87.2 \%$, bis-1,1'-(1methyl-1,2-ethanediyl) Benzene $\left(\mathrm{C}_{15} \mathrm{H}_{16}\right)(\mathrm{t}=16.89, \mathrm{~m} / \mathrm{z}=105)$ Compound probability percentage is $39.0 \%$, Hexadecane $\left(\mathrm{C}_{16} \mathrm{H}_{34}\right)(\mathrm{t}=17.24, \mathrm{~m} / \mathrm{z}=57)$ Compound probability percentage is $9.89 \%$, 1,1'-ethylidenebis- Benzene $\left(\mathrm{C}_{14} \mathrm{H}_{14}\right) \quad(\mathrm{t}=17.43, \mathrm{~m} / \mathrm{z}=167)$ Compound probability percentage is $36.9 \%$, bis-1,1'-(1-methyl-1,3-propanediyl)Benzene $\left(\mathrm{C}_{16} \mathrm{H}_{18}\right)(\mathrm{t}=18.43, \mathrm{~m} / \mathrm{z}=105)$ Compound probability percentage is $90.5 \%$, bis-1,1'-(3methyl-1-propene-1,3-diyl) Benzene $\left(\mathrm{C}_{16} \mathrm{H}_{16}\right) \quad(\mathrm{t}=19.73, \mathrm{~m} / \mathrm{z}=115) \quad$ Compound probability percentage is $48.1 \%$, trans- (2,3-Diphenylcyclopropyl)methyl phenyl sulfoxide $\left(\mathrm{C}_{22} \mathrm{H}_{20} \mathrm{OS}\right)(\mathrm{t}=25.80, \mathrm{~m} / \mathrm{z}=91)$ Compound probability percentage is $26.5 \%$. Analysis result indicates that product fuel has high percentage of aromatic group then aliphatic group.

\section{Conclusion}

Motor vehicle waste tire and polystyrene waste plastic mixture to fuel production was performed without catalyst at $100-420{ }^{\circ} \mathrm{C}$. Product fuel is ignited and fuel was analysis by GC/MS to checked fuel inside compounds structure. Carbon chain 
showed $\mathrm{C}_{3}-\mathrm{C}_{22}$ and most of the compounds are aromatic group including Benzene, Toluene, Ethylbenzene, p-Xylene, 2-propenyl-Benzene, $\alpha$-Methylstyrene, 2-propenylBenzene, Limonene, Indane, 1-methyl-4-(2-propenyl)-Benzene, Naphthalene, 3cyclohexen-1-yl-Benzene, bis-1,1'-(1,2-ethanediyl)Benzene, 1-methyl-3-(phenylmethyl)Benzene, bis-1,1'-(1-butenylidene)Benzene, and so on. PS waste plastic has aromatic group and motor vehicle has also aromatic group compounds. Liquid fuel conversion rate only $54.93 \%$ and rest of percentage was solid residue and light gas. Residue percentage was high because tire has high percentage of additive, cloth, rubber. Rubber, cloth and additives that not convertible into fuel. This technology can convert all PS waste plastic and motor vehicle used tire into useful chemicals which can use internal combustion engines and refinery process for feed.

\section{Acknowledgement}

The authors acknowledge the support [financial] of Dr. Karin Kaufman, the founder and sole owner of Natural State Research, Inc. The authors also acknowledge the valuable contributions NSR laboratory team members during the preparation of this manuscript.

\section{References}

[1] Larsen, M. B.; Schultz, L.; Glarborg, P.; Skaarup-Jensen, L.; Dam- Johansen, K.; Frandsen, F.; Herriksen, U. Devolatilization characteristics of large particles of tyre rubber under combustion conditions. Fuel 2006, 85, 1335-1345.

[2] Sunthonpagasit, N.; Duffey, R. Scrap tires to crumb rubber: feasibility analysis for processing facilities. Resour., ConserV. Recycl. 2004, 40, 281-299.

[3] Huffman, G. P.; Shah, N. Can waste plastics and tires be recycled economically. CHEMTECH 1998, 28, 34-43.

[4] Sharma, V. K.; Fortuna, F.; Macarini, M.; Berillo, M.; Cornacchia, G. Disposal of waste tyres for energy recovery and safe environment. Appl. Energy 2000, 65, 381-394.

[5] de Marco, I.; Laresgoiti, M. F.; Cabrero, M. A.; Torres, A.; Chomon, M. J.; Caballero, B. Pyrolysis of scrap tyres. Fuel Process. Technol. 2001, 72, 9-22.

[6] Gonzalez, J. F.; Encinar, J. M.; Canito, J. L.; Rodriguez, J. J. Pyrolysis of automobile tyre waste. Influence of operating variables and kinetics study. J. Anal. Appl. Pyrolysis 2001, 58, 667-683.

[7] Laresgoiti, M. F.; de Marco, I.; Torres, A.; Caballero, B.; Cabrero, M. A.; Chomon, M. J. Chromatographic analysis of the gases obtained in tyre pyrolysis. J. Anal. Appl. Pyrolysis 2000, 55, 43-54.

[8] Laresgoiti, M. F.; Caballero, B.; de Marco, I.; Torres, A.; Cabrero, M. A.; Chomo'n, M. J. Characterization of the liquid products obtained in tyre pyrolysis. J. Anal. Appl. Pyrolysis 2004, 71, 917-934.

[9] Berrueco, C.; Esperanza, E.; Mastral, F. J.; Ceamanos, J.; Garcia- Bacaicoa, P. Pyrolysis of waste tyres in an atmospheric static-bed batch reactor: Analysis of the gases obtained. J. Anal. Appl. Pyrol. 2005, 74, 245- 253.

[10] Ucar, S.; Karagoz, S.; Ozkan, A. R.; Yanik, J. Evaluation of two different scrap tires as hydrocarbon source by pyrolysis. Fuel 2005, 84, 1884-1892. 


\section{Sarker and M. M. Rashid}

[11] Williams, P. T.; Besler, S.; Taylor, D. T. The pyrolysis of scrap automotive tyres: The influence of temperature and heating rate on product composition. Fuel 1990, 69, 1474 1482.

[12] Lee, J. M.; Lee, J. S.; Kim, J. R.; Kim, S. D. Pyrolysis of waste tires with partial oxidation in a fluidized-bed reactor. Energy 1995, 20, 969- 976.

[13] Wey, M. Y.; Huang, S. C.; Shi, C. L. Oxidative pyrolysis of mixed solid wastes by sand bed and freeboard reaction in a fluidized bed. Fuel 1997, 76, 115-121.

[14] Kaminsky, W.; Mennerich, C. Pyrolysis of synthetic tire rubber in a fluidised-bed reactor to yield 1,3-butadiene, styrene and carbon black. J. Anal. Appl. Pyrolysis 2001, 58-59, 803811.

[15] Roy, C.; Labrecque, B.; de Caumia, B. Recycling of scrap tires to oil and carbon black by vacuum pyrolysis. Resour., ConserV. Recycl. 1990, 51, 203-213.

[16] Roy, C.; Chaala, A.; Darmstadt, H. The vacuum pyrolysis of used tires: End-uses for oil and carbon black products. J. Anal. Appl. Pyrolysis 1999, 51, 201-221.

[17] Benallal, B.; Roy, C.; Pakdel, H.; Chabot, S.; Porier, M. A. Characterization of pyrolytic light naphtha from vacuum pyrolysis of used tyres comparison with petroleum naphtha. Fuel 1995, 74, 1589-1594.

[18] Bridgwater, A. V.; Peacocke, G. V. C. Fast pyrolysis processes for biomass. Renewable Sustainable Energy ReV. 2000, 4, 1-73.

[19] Fortuna, F.; Cornacchia, G.; Mincarini, M.; Sharma, V. K. Pilotscale experimental pyrolysis plant: Mechanical and operational aspects. J. Anal. Appl. Pyrolysis 1997, 40-41, 403-417, May 1997.

[20] Li, S. Q.; Yao, Q.; Chi, Y.; Yan, J. H.; Cen, K. F. Pilot-scale pyrolysis of scrap tires in a continuous rotary kiln reactor. Ind. Eng. Chem. Res. 2004, 43, 5133-5145.

[21] Diez, C.; Sanchez, M. E.; Haxaire, P.; Martınez, O.; Moran, A. Pyrolysis of tyres: A comparison of the results from a fixed-bed laboratory reactor and a pilot plant (rotary reactor). J. Anal. Appl. Pyrolysis 2005, 74, 254-258.

[22]Miriam Arabiourrutia, Martin Olazar, Roberto Aguado, Gartzen Lopez, Astrid Barona, and Javier Bilbao, HZSM-5 and HY Zeolite Catalyst Performance in the Pyrolysis of Tires in a Conical Spouted Bed Reactor, Ind. Eng. Chem. Res. 2008, 47, 7600-7609

[23] S.-Q. Li, Q. Yao, Y. Chi, J.-H. Yan, and K.-F. Cen, Pilot-Scale Pyrolysis of Scrap Tires in a Continuous Rotary Kiln Reactor, Ind. Eng. Chem. Res. 2004, 43, 5133-5145

[24] Kaminsky, W.; Mennerich, D. Pyrolysis of synthetic tire rubber in a fluidised-bed reactor to yield 1,3-butadiene, styrene and carbon black. J. Anal. Appl. Pyrolysis 2001, 58-59, 803.

[25] Roy, C.; Chaala, A.; Darmstadt, H. The vacuum pyrolysis of used tires End-uses for oil and carbon black products. J. Anal. Appl. Pyrolysis 1999, 51, 201.

[26] Bilitewaki, B.; Ha“" rdtle, G.; Marek, K. Usage of carbon black and activated carbon in relation to input and technical aspects of the pyrolysis process. In Pyrolysis and gasification; Ferrero, G. L., Maniatis, K., Buekens, A., Eds.; Elsevier Applied Science: London, U.K., 1989.

[27] San Miguel, G.; Fowler, G. D.; Sollars, C. J. Pyrolysis of tire rubber: porosity and adsorption characteristics of pyrolytic chars. Ind. Eng. Chem. Res. 1998, 37, 2430.

[28] Teng, H.; Serio, M. A.; Wojtowicz, M. A.; Bassilakis, R.; Solomon, P. R. Reprocessing of used tires into activated carbon and other products. Ind. Eng. Chem. Res. 1995, 34, 3102.

[29] Merchant, A. A.; Petrich, M. A. Pyrolysis of scrap tires and conversion of chars to activated carbon. AIChE J. 1993, 39, 1370.

[30] Collins, L. W.; Downs, W. R.; Gibson, L. K.; Moore, G. W. An evalation of discarded tires as source of fuel. Thermochim. Acta 1974, 10, 153. 
[31] De Marco Rodriguez, I.; Laresgoiti, M. F.; Cabrero, M. A.; Torres, A.; Chmon, M. J.; Caballero, B. Pyrolysis of scrap tyres. Fuel Process Technol. 2001, 72, 9.

[32] Williams, P. T.; Bottrill, R. P.; Cunliffe, A. M. Combustion of tyre pyrolysis oil. Trans. Inst. Chem. Eng. 1998, 76B, 291.

[33] Williams, P. T.; Besler, S.; Taylor, D. T. The batch pyrolysis of tyre wastesfuel properties of the derived oils and overall plant economics. Proc. Inst. Mech. Eng. 1993, $207,55$.

[34] Benallal, B.; Roy, C.; Pakdel, H.; Chabot, S.; Poirier, M. A. Characterisation of pyrolytic light naphtha from vacuum pyrolysis of used tyres. Comparison with petroleum naphtha. Fuel 1995, 74, 1589.

[35] Pakdel, H.; Roy, C. Simultaneous gas chromatographic- Fourier transform infrared spectroscopic-mass spectrometric analysis of synthetic fuel derived from used tire pyrolysis oil, naphtha fraction. J. Chromatogr. A 1994, 683, 203.

[36] Pakdel, H.; Pantea, D. M.; Roy, C. Production of dllimonene by vacuum pyrolysis of used tires. J. Anal. Appl. Pyrolysis 2001, 57, 91.

[37] Pakdel, H.; Roy, C.; Aubin, H.; Jean, G.; Coulombe, S. Formation of DL-limonene in used tire vacuum pyrolysis oils. Environ. Sci. Technol. 1992, 25, 1646.

[38] Cypres, R.; Bettens, B. Production of Benzoles and Active Carbon from Waste Rubber and Plastic Materials by Means of Pyrolysis with Simultaneous Post-cracking. In Pyrolysis and gasification; Ferrero, G. L., Maniatis, K., Buekens, A., Eds.; Elsevier Applied Science: London, U.K., 1989.

[39] Kaminsky, W.; Shin, H. Pyrolysis of plastic waste and scrap tyres using a fluidised bed process. In Thermal Conversion of Solid Waste and Biomass; Jones, J. L., Radding, S. B., Eds.; ACS Symposium Series 130; American Chemical Society: Washington, DC, 1980; p 423.

[40] Kaminsky, W.; Kim, J.-s. Pyrolysis of mixed platics into aromatics. J. Anal. Appl. Pyrolysis $1999,51,127$.

[41] Williams, P. T.; Taylor, D. T. Aromatisation of tyre pyrolysis oil to yield polycylic aromatic hydrocarbons. Fuel 1993, 72, 1469.

[42] Williams, P. T.; Bottrill, R. P. Sulfur-polycylic aromatic hydrocarbons in tyre pyrolysis. Fuel 1995, 74, 736.

[43] Williams, P. T.; Besler, S.; Taylor, D. T. The pyrolysis of scrap automotive tyres: the influence of temperature and heating rate on product combustion. Fuel 1990, 69, 1474.

[44] Williams, P. T.; Besler, S.; Taylor, D. T.; Bottrill, R. P. Pyrolysis of automotive tyre waste. J. Inst. Energy 1995, 68, 11.

[45] Roy, C.; Labrecque, B.; de Caumia, B. Recycling of scrap tyres to oil and carbon black by vacuum pyrolysis. Resour., Conserv. Recycl. 1990, 4, 203. 